\title{
Effets combinés de la nisine, de l'acide lactique et du sorbate de potassium sur les spores de Bacillus licheniformis dans le lait
}

\author{
Marianne Mansour ${ }^{\mathrm{a}}$, Michel Linder ${ }^{\mathrm{b}}$, Jean-Bernard Millière ${ }^{\mathrm{a}}$, \\ Gérard Lefebvre ${ }^{a}$
}

${ }^{a}$ Laboratoire de fermentations et bioconversions industrielles,

${ }^{b}$ Laboratoire de physico-chimie et génie alimentaires, Ensaia-INPL, 2, avenue de la Forêt-de-Haye, 54500 Vandouvre-lès-Nancy, France

\begin{abstract}
Combined effects of nisin, lactic acid, and potassium sorbate on Bacillus licheniformis spores in milk. The inhibitory activities of nisin and/or potassium sorbate on Bacillus licheniformis spores were studied in milk acidified with lactic acid, at $37^{\circ} \mathrm{C}$. Three experimental plans were realized using the Doehlert uniform shell design. Spore germination, $\mathrm{pH}$-dependent, was enhanced as the $\mathrm{pH}$ increased. Nisin had no sporicidal effect, but inhibited the growth of germinated spores. Its bactericidal activity increased with the concentration of nisin and as the $\mathrm{pH}$ and the initial spore load decreased. Potassium sorbate was not sporicidal but disturbed the spore germination at high concentrations and low $\mathrm{pH}$ values. Its bactericidal effect was favoured at acidic $\mathrm{pH}$ values. Nisin and sorbate potassium in association enhanced the bactericidal effect with an important inhibition at high $\mathrm{pH}$ values. (0) Inra/Elsevier, Paris.
\end{abstract}

Bacillus licheniformis / spore / nisin / potassium sorbate / lactic acid

Résumé - Les activités inhibitrices de la nisine et/ou du sorbate de potassium sur les spores de Bacillus licheniformis sont étudiées dans du lait acidifié avec de l'acide lactique, à $37^{\circ} \mathrm{C}$. Trois plans d'expériences sont réalisés selon les réseaux uniformes de Doehlert. La germination des spores, $\mathrm{pH}$-dépendante, est favorisée aux valeurs proches de la neutralité. La nisine, non sporicide, est bactéricide sur les spores germées ; cette activité augmente avec la concentration en nisine, lorsque le $\mathrm{pH}$ et la concentration initiale en spores diminuent. Le sorbate de potassium, sans action sporicide, retarde la germination aux concentrations élevées et à pH acide. Son activité bactéricide est favorisée à $\mathrm{pH}$ acide. L'association de la nisine et du sorbate de potassium augmente le pouvoir bactéricide avec une inhibition maximale aux $\mathrm{pH}$ proches de la neutralité. (c) Inra/Elsevier, Paris.

Bacillus licheniformis / spore / nisine / sorbate de potassium / acide lactique 


\section{INTRODUCTION}

La présence de bactéries sporulées (Clostridium, Bacillus) dans les aliments est à l'origine d'altérations et parfois d'intoxications alimentaires graves. Leur aptitude à élaborer des spores, organites de résistance, nécessite des traitements technologiques spécifiques tels qu'appertisation, bactofugation ou ajout de conservateurs chimiques, afin d'augmenter la durée de vie des aliments. Le genre Bacillus, ubiquitaire, contamine le lait cru lors de l'utilisation d'ensilages et/ou d'équipements de traite de mauvaise qualité hygiénique. En France, dans le genre Bacillus, B. licheniformis est fréquemment mentionné à des taux variant entre 10 et $50 \%$ [5]. Malgré une hygiène de plus en plus rigoureuse à tous les stades de la production et de la transformation, les niveaux de contamination en $B$. licheniformis, de 10 à $100 \mathrm{UFC} / \mathrm{mL}$ [27], restent encore relativement importants. Cette espèce peut être à l'origine de mammites bovines [7] ou quelquefois de méningites humaines [10]. Dans les fromages fabriqués avec du lait pasteurisé, la présence de $B$. licheniformis peut provoquer une protéolyse importante [12].

Sous la pression des consommateurs, les industriels sont progressivement contraints de réduire l'emploi des conservateurs chimiques au profit d'inhibiteurs naturels assurant sécurité et qualité alimentaires. La nisine, polypeptide antibactérien, est produite par certaines espèces de Lactococcus lactis subsp. lactis ; elle est la seule bactériocine autorisée comme additif alimentaire (E 234) depuis 1969 (OMS). De statut GRAS (generally recognized as safe), elle possède un large spectre d'activité antibactérienne, essentiellement dirigé contre les bactéries Gram positives. En 1951, la nisine fut préconisée par Hirsch [11], afin d'éviter le gonflement de fromages à pâte pressée cuite par Clostridium tyrobutyri- cum ; elle fut également utilisée dans la conservation de fromages fondus [20], pour réduire ou même supprimer l'emploi de conservateurs chimiques, comme les nitrites [23]. Le sorbate de potassium (E 202), également de statut GRAS, est autorisé aux États-Unis dans tous les produits alimentaires nécessitant l'ajout de conservateurs [18]. Utilisé comme antifongique, son emploi peut être envisagé contre diverses bactéries pathogènes ou des spores [3, 21, 26], dans les industries alimentaires, pharmaceutiques, cosmétiques ou dans les emballages.

L'objectif de ce travail est d'étudier le comportement des spores de B. licheniformis dans du lait acidifié par ajout d'acide lactique, en présence de nisine et/ou de sorbate de potassium. En microbiologie alimentaire, l'utilisation de modèles mathématiques fait l'objet de nombreux travaux, afin de prédire le comportement des microorganismes dans diverses conditions environnementales [1, 2]. Nos expérimentations sont effectuées suivant un modèle mathématique utilisant les réseaux uniformes de Doehlert [9]. Trois plans d'expérience sont retenus : (i) effets de la nisine et du $\mathrm{pH}$ sur la germination et sur l'inhibition de la croissance des spores germées, (ii) effets du sorbate et du $\mathrm{pH}$, (iii) effets de la nisine, du sorbate de potassium et du $\mathrm{pH}$. Les effets des différents facteurs et de leurs interactions permettent d'optimiser les résultats, à partir de la méthodologie des surfaces de réponse.

\section{MATÉRIELS ET MÉTHODES}

\subsection{Microorganisme}

Bacillus licheniformis ATCC 14580 est retenu dans toutes les expérimentations. Après conservation à $4{ }^{\circ} \mathrm{C}$ sur tryptone agar (Difco), la souche est repiquée en bouillon IP (Institut Pasteur) de composition (en g/L) : bactopeptone (Difco) 6 ; tryptone (Biokar) 4 ; extrait de 
levure (Biokar) 3 ; extrait de viande bactériologique (Biokar) 1,5; glucose (Biokar) 1 ; $\mathrm{pH} 6,75$.

\subsection{Préparation des spores}

Les spores sont récoltées après culture dans le bouillon de sporulation A5 [16] de composition en $\mathrm{g} / \mathrm{L}$ : glucose, 3,6 ; lactate d'ammonium, 5,$4 ; \mathrm{HNO}_{3}, 0,31 ; \mathrm{H}_{3} \mathrm{PO}_{4}, 0,31 ; \mathrm{NaCl}$, 0,$4 ; \mathrm{MgSO}_{4}, 7 \mathrm{H}_{2} 0,6 \mathrm{mg} ;\left(\mathrm{NH}_{4}\right)_{2} \mathrm{SO}_{4}, 6 \mathrm{H}_{2} \mathrm{O}$, $25 \mathrm{mg} ; \mathrm{pH} 7,2$ ajusté avec $\mathrm{KOH} 1 \mathrm{~N}$. Ce bouillon est ensemencé à $10 \%(\mathrm{v} / \mathrm{v})$ à partir d'une préculture de 24 heures à $37^{\circ} \mathrm{C}$ en bouillon IP. Après incubation à $37^{\circ} \mathrm{C}$ pendant 7 jours, les spores sont récoltées par centrifugation $(8000 \mathrm{~g}, 10 \mathrm{~min})$ puis remises en suspension dans $20 \mathrm{~mL}$ d'eau distillée désionisée. La suspension de spores est chauffée à $80^{\circ} \mathrm{C}$ pendant 10 minutes pour détruire les cellules végétatives, puis conservée à $4^{\circ} \mathrm{C}$ jusqu'à son utilisation, après numération cellulaire (2.10 $0^{8}$ spores $\left./ \mathrm{mL}\right)$.

\subsection{Inhibiteurs}

La solution mère de nisine $\left(10^{4} \mathrm{UI} / \mathrm{mL}\right.$ : Nisin ${ }^{\circledR}$ Sigma) est préparée dans $\mathrm{HCl} \mathrm{0,02} \mathrm{N}$. La solution mère de sorbate de potassium $(4 \%$ $\mathrm{p} / \mathrm{v}$; Sigma) en eau distillée est filtrée stérilement sur filtre de porosité $0,22 \mu \mathrm{m}$ (OSI).

\subsection{Numérations des cellules totales et des spores}

Les numérations des cellules totales et des spores après chauffage $\left(80^{\circ} \mathrm{C}, 10 \mathrm{~min}\right)$ sont effectuées en bouillon IP gélosé avec $15 \mathrm{~g} / \mathrm{L}$ d'agar bactériologique (Biokar). Les dilutions décimales sont réalisées en bouillon tryptone sel (Biokar) à 9,5 g/L. Après ensemencement en profondeur avec $1 \mathrm{~mL}$ d'échantillon, une fine couche de gélose blanche $(5 \mathrm{~mL})$ est coulée en surface pour empêcher son envahissement par les colonies de Bacillus. La germination des spores est mesurée par la perte de leur thermorésistance. La numération des cellules végétatives correspond à la différence entre les dénombrements des cellules totales et des spores. Le pourcentage de germination, selon la formule d'Irie et al. [14], correspond à [(spores initiales - spores finales) / spores initiales $] \times 100$.

\subsection{Activités inhibitrices de la nisine et du sorbate de potassium}

Les modes d'action de ces deux inhibiteurs sont étudiés dans du lait écrémé (Oxoid) à $10 \%$ $(\mathrm{p} / \mathrm{v})$, acidifié avec de l'acide lactique ( $99 \%$, Prolabo). Le lait est supplémenté avec $1 \mathrm{~g} / \mathrm{L}$ de L-alanine (Sigma), comme promoteur de germination. Les spores sont ajoutées après une activation thermique à $80^{\circ} \mathrm{C}$ pendant 10 minutes. Les inhibiteurs sont ensuite introduits aux différentes concentrations retenues. L'incubation est réalisée à $37{ }^{\circ} \mathrm{C}$ et des prélèvements sont effectués à 6 heures ou à 24 heures pour dénombrer les cellules totales et les spores et pour déterminer le $\mathrm{pH}$. La nisine présente dans l'échantillon est inactivée par ajout de $1 \mathrm{mg} / \mathrm{mL}$ de protéase de type XIV de Streptomyces griseus (Sigma). L'effet inhibiteur du sorbate est supprimé en neutralisant l'échantillon à $\mathrm{pH} 7,0$ avec $\mathrm{NaOH} \mathrm{N}$.

\subsection{Réseaux uniformes de Doehlert}

Les activités inhibitrices de la nisine et du sorbate de potassium ont été étudiées en fonction du $\mathrm{pH}$ et des concentrations en spores, de manière indépendante (tableau I) ou en combinaison (tableau II), en postulant un modèle mathématique empirique. L'estimation des coefficients du modèle quadratique prévisionnel est obtenue à partir d'un plan d'expérimentations construit selon les réseaux uniformes de Doehlert. Cette matrice, générée à partir d'un simplex, présente une distribution uniforme des essais sur l'ensemble du domaine expérimental et permet d'attribuer différents niveaux aux variables indépendantes en fonction de leur importance (tableau III). Seize expériences ont été réalisées $\left(\mathrm{N} \geq \mathrm{k}^{2}+\mathrm{k}+1\right)$ avec trois répétitions de l'essai au centre du domaine, afin de prendre en compte l'erreur expérimentale. Les résultats expérimentaux et la prédiction des réponses sur l'ensemble du domaine expérimental peuvent être visualisés sous forme de courbes d'isopopulation générées par le logiciel NEMROD ${ }^{\circledR}$ [19]. Il est ainsi possible de suivre l'évolution de chacune des réponses en fixant un paramètre, généralement au centre du domaine. Le domaine expéri- 
Tableau I. Domaine expérimental et niveaux des facteurs retenus pour l'évaluation des activités inhibitrices de la nisine et du sorbate de potassium en fonction du pH et de la concentration en spores.

Table I. Experimental domain and levels of retained factors for the evaluation of the inhibitory activities of nisin and potassium sorbate as a function of $\mathrm{pH}$ and spore concentration.

\begin{tabular}{|c|c|c|c|}
\hline & Facteurs & Niveaux & Valeurs expérimentales \\
\hline \multirow{4}{*}{ ou } & $\mathrm{X} 1$ : nisine $(\mathrm{UI} / \mathrm{mL})$ & 5 & $0-10-20-30-40$ \\
\hline & $\mathrm{X} 1:$ sorbate $(\% \mathrm{p} / \mathrm{v})$ & 5 & $0,00-0,05-0,10-0,15-0,20$ \\
\hline & $\mathrm{X} 2: \mathrm{pH}$ & 7 & $5,0-5,2-5,4-5,6-5,8-6,0-6,2$ \\
\hline & $\mathrm{X} 3$ : spores $/ \mathrm{mL}$ & 3 & $10^{4}-10^{5}-10^{6}$ \\
\hline
\end{tabular}

Tableau II. Domaine expérimental et niveaux des facteurs retenus pour l'évaluation des activités inhibitrices de la nisine et du sorbate de potassium, en fonction du $\mathrm{pH}$. La concentration initiale en spores est de $10^{5}$ spores $/ \mathrm{mL}$.

Table II. Experimental domain and levels of retained factors for the evaluation of the inhibitory activities of nisin and potassium sorbate as a function of $\mathrm{pH}$ values. The initial spore load is $10^{5}$ spores $/ \mathrm{mL}$.

\begin{tabular}{lcc}
\hline Facteurs & Niveaux & Valeurs expérimentales \\
\hline $\mathrm{X} 1:$ nisine $(\mathrm{UI} / \mathrm{mL})$ & 5 & $0-10-20-30-40$ \\
$\mathrm{X} 2: \mathrm{pH}$ & 7 & $5,0-5,2-5,4-5,6-5,8-6,0-6,2$ \\
$\mathrm{X} 3:$ sorbate $(\% \mathrm{p} / \mathrm{v})$ & 3 & $0,0-0,1-0,2$ \\
\hline
\end{tabular}

Tableau III. Plan des essais expérimentaux pour trois variables (X1, X2, X3) comprenant trois répétitions au centre du domaine expérimental (essais 13a, 13b,13c) selon les réseaux uniformes de Doehlert [9]. Les valeurs sont exprimées en variables centrées réduites.

Table III. Experimental design for three variables (X1, X2, X3) including three repetitions at the center of the domain $(13 \mathrm{a}, 13 \mathrm{~b}, 13 \mathrm{c})$ according to Doehlert uniform shell design [9]. Values are expressed in coded variables.

\begin{tabular}{lrrr}
\hline Essais & $\mathrm{X} 1$ & $\mathrm{X} 2$ & $\mathrm{X} 3$ \\
\hline 1 & 1,000 & 0,000 & 0,000 \\
2 & $-1,000$ & 0,000 & 0,000 \\
3 & 0,500 & 0,866 & 0,000 \\
$13 \mathrm{a}$ & 0,000 & 0,000 \\
4 & $-0,500$ & $-0,866$ & 0,000 \\
5 & 0,500 & $-0,866$ & 0,000 \\
6 & $-0,500$ & 0,866 & 0,000 \\
$13 \mathrm{~b}$ & 0,000 & 0,000 & 0,000 \\
7 & 0,500 & 0,289 & 0,816 \\
8 & $-0,500$ & $-0,289$ & $-0,816$ \\
9 & 0,500 & $-0,289$ & $-0,816$ \\
$13 \mathrm{c}$ & 0,000 & 0,000 & 0,000 \\
10 & 0,000 & 0,577 & $-0,816$ \\
11 & $-0,500$ & 0,289 & $-0,816$ \\
12 & 0,000 & $-0,577$ & $-0,816$ \\
13 & 0,000 & 0,000 & 0,000 \\
\hline
\end{tabular}


mental est défini à partir d'expérimentations antérieures et les réponses mesurées pour les trois plans d'expériences sont les nombres de spores et les nombres de cellules végétatives à $6 \mathrm{~h}$ et à $24 \mathrm{~h}$.

\section{RÉSULTATS}

Chacune des réponses peut être représentée par un modèle quadratique, comprenant 10 coefficients, valable sur l'ensemble du domaine expérimental :

$$
\begin{aligned}
\eta_{j}= & B_{j 0}+\sum_{i=1}^{3} B_{j i j} X_{i}+\sum_{i=1}^{3} B_{j i i} X_{i}^{2} \\
& +\sum_{i=1}^{2} \sum_{j=i+1}^{3} B_{j i j} X_{i} X_{j}
\end{aligned}
$$

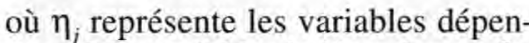
dantes, $X_{i}$ les variables indépendantes, $\beta_{j 0}$ le terme constant, $\beta_{j i}$ les coefficients linéaires du premier ordre, $\beta_{j \mathrm{ii}}$ les termes carrés, $\beta_{j i j}$ les termes d'interaction. Ces derniers permettront de quantifier et de mieux caractériser les activités inhibitrices de la nisine et du sorbate de potassium. Les valeurs sont reportées dans les tableaux IV (spores) et V (cellules végétatives).

\subsection{Combinaison nisine / $\mathrm{pH}$}

\subsubsection{Sur les spores}

Les estimations des effets des différentes variables montrent que la nisine n'exerce pas d'activité inhibitrice significative sur les spores et n'empêche pas la germination, qui est fortement dépendante du $\mathrm{pH}$ (figure 1 a); le pourcentage de germination calculé est de $94 \%$ à pH 5,6, en présence de 0,20 ou de $40 \mathrm{UI} / \mathrm{mL}$ de nisine. Entre 5,0 et 6,2, les niveaux des courbes d'isopopulation diminuent quand le $\mathrm{pH}$ augmente, en relation avec la ger-

Tableau IV. Estimation des coefficients des différents modèles quadratiques postulés pour la réponse : nombre de spores à 6 ou 24 heures en présence de nisine et/ou de sorbate de potassium.

Table IV. Coefficient estimation of different quadratic models postulated for the response: spore

\begin{tabular}{|c|c|c|c|c|c|c|}
\hline \multirow{3}{*}{$\begin{array}{l}\text { Estimation } \\
\text { des } \\
\text { coefficients }\end{array}$} & \multicolumn{6}{|c|}{ Durées d'incubation } \\
\hline & \multicolumn{3}{|c|}{$6 \mathrm{~h}$} & \multicolumn{3}{|c|}{$24 \mathrm{~h}$} \\
\hline & Nisine & Sorbate & $\begin{array}{c}\text { Nisine+ } \\
\text { sorbate }\end{array}$ & Nisine & Sorbate & $\begin{array}{l}\text { Nisine+ } \\
\text { sorbate }\end{array}$ \\
\hline$\beta 0$ & $3,7413_{\mathrm{a}}$ & $3,9933_{\mathrm{a}}$ & $3,8100_{\mathrm{a}}$ & 1,9832 a & $2,6968_{a}$ & $2,2143_{\mathrm{a}}$ \\
\hline$\beta 1$ & $0,0159^{\circ}$ & $0,2121_{\mathrm{a}}{ }^{a}$ & $-0,0345^{\mathrm{a}}$ & $-0,6089^{a}$ & $0,2186_{b}$ & $0,0188^{\mathrm{a}}$ \\
\hline$\beta 2$ & $-1,0145_{a}$ & $-1,1425_{a}^{a}$ & $-0,9617_{\mathrm{a}}$ & $-1,3613_{a}^{a}$ & $-1,1877_{\mathrm{a}}$ & $-1,6218_{a}$ \\
\hline$\beta 3$ & $1,3578_{\mathrm{a}}$ & $1,0643_{\mathrm{a}}$ & $0,2064_{b}$ & $1,4509_{\mathrm{a}}$ & $0,8495_{\mathrm{a}}$ & $0,3633_{\mathrm{a}}$ \\
\hline$\beta 11$ & $0,1608_{c}$ & $0,2407_{b}$ & $0,1950_{\mathrm{c}}$ & $1,0233_{\mathrm{a}}$ & $0,2072_{\mathrm{c}}$ & 0,0853 \\
\hline$\beta 22$ & $0,3621_{\mathrm{a}}$ & $0,2157_{\mathrm{b}}$ & $0,2120_{\mathrm{c}}$ & $0,9359_{\mathrm{a}}$ & $1,1915_{\mathrm{a}}$ & $0,9589_{\mathrm{a}}$ \\
\hline$\beta 33$ & $0,1474_{c}$ & $0,4095_{\mathrm{a}}$ & $-0,0870$ & 0,0923 & $0,8110_{a}$ & $0,2236_{c}$ \\
\hline$\beta 12$ & $-0,0046$ & $0,1288_{\mathrm{c}}$ & 0,1241 & $-0,7044 b$ & $-0,9474 \mathrm{a}$ & $-0,3585_{c}$ \\
\hline$\beta 13$ & $-0,0357$ & 0,0377 & 0,2151 & 0,2533 & 0,0985 & 0,1862 \\
\hline \multirow[t]{2}{*}{$\beta 23$} & $\begin{array}{c}-0,0833 \\
R^{2}=0,99\end{array}$ & $\begin{array}{c}-0,0061 \\
\mathrm{R}^{2}=0.99\end{array}$ & $\begin{array}{c}0,2814 \\
R^{2}=0,99\end{array}$ & $\begin{array}{c}-0,3091 \\
R^{2}=0.95\end{array}$ & $\begin{array}{l}-0,8301 \\
R^{2}=0.95\end{array}$ & $\begin{array}{c}-0,3660^{c} \\
R^{2}=0.99\end{array}$ \\
\hline & $\mathrm{R}^{2} \mathrm{~A}=0,99$ & $\mathrm{R}^{2} \mathrm{~A}=0,99$ & $\mathrm{R}^{2} \mathrm{~A}=0,97$ & $\mathrm{R}^{2} \mathrm{~A}=0,88$ & $\mathrm{R}^{2} \mathrm{~A}=0,89$ & $\mathrm{R}^{2} \mathrm{~A}=0,98$ \\
\hline
\end{tabular}
count after 6 or $24 \mathrm{~h}$ with nisin and/or potassium sorbate.

a: $\alpha \leq 1 \%_{0} ;$ b : $1 \%_{0}<\alpha \leq 1 \% ; c: 1 \%<\alpha \leq 5 \%$. 
Tableau V. Estimation des coefficients des différents modèles quadratiques postulés pour la réponse : nombre de cellules végétatives à 6 ou 24 heures en présence de nisine et/ou sorbate de potassium.

TableV. Coefficient estimation of different quadratic models postulated for the response: vegetative cell count after 6 or $24 \mathrm{~h}$ with nisin and/or potassium sorbate.

\begin{tabular}{|c|c|c|c|c|c|c|}
\hline \multirow{3}{*}{$\begin{array}{l}\text { Estimation } \\
\text { des } \\
\text { coefficients }\end{array}$} & \multicolumn{6}{|c|}{ Durées d'incubation } \\
\hline & \multicolumn{3}{|c|}{$6 \mathrm{~h}$} & \multicolumn{3}{|c|}{$24 \mathrm{~h}$} \\
\hline & Nisine & Sorbate & $\begin{array}{c}\text { Nisine+ } \\
\text { sorbate }\end{array}$ & Nisine & Sorbate & $\begin{array}{l}\text { Nisine+ } \\
\text { sorbate }\end{array}$ \\
\hline$\beta 0$ & $3,3138_{\mathrm{a}}$ & 5,2564 & 3,9340 & $6,3010_{\mathrm{a}}$ & 7,2222 & 2,5160 \\
\hline$\beta 1$ & $-2,0209_{b}^{a}$ & $-0,3509^{a}$ & $-1,5388_{a}^{a}$ & $-2,6649^{a}$ & $-0,5514^{a}$ & $-2,6866^{a}$ \\
\hline$\beta 2$ & $0,4072^{\circ}$ & $2,8532_{\mathrm{a}}^{\mathrm{a}}$ & $-1,0434_{a}^{a}$ & $3,1720_{b}^{c}$ & $3,8648_{a}^{c}$ & $0,2167_{\mathrm{c}}^{\mathrm{a}}$ \\
\hline$\beta 3$ & $1,8200_{b}$ & $1,1105_{\mathrm{a}}$ & $0,3991_{b}^{a}$ & $2,0925_{\mathrm{c}}^{\circ}$ & $1,0501_{b}^{a}$ & $-1,6920_{\mathrm{a}}$ \\
\hline$\beta 11$ & 1,0768 & $0,4906_{a}$ & $-1,2700_{a}$ & $-2,67575$ & $-1,4147 \mathrm{c}$ & $1,0085_{b}$ \\
\hline$\beta 22$ & $-1,0329$ & $-2,9804_{a}$ & $0,4917_{\mathrm{c}}^{\mathrm{a}}$ & $-3,1142^{\mathrm{c}}$ & $-4,2244_{a}$ & $3,2182_{\mathrm{a}}^{\mathrm{D}}$ \\
\hline$\beta 33$ & 0,8669 & $0,4075_{b}$ & 0,2088 & $-0,5608$ & $-0,7434$ & $3,5211_{\mathrm{a}}$ \\
\hline$\beta 12$ & $1,8897_{\mathrm{c}}$ & $-0,2997_{\mathrm{c}}$ & $-0,5802_{c}$ & $-0,3476$ & $-0,0930$ & $-2,0225_{a}$ \\
\hline$\beta 13$ & $-1,4078$ & 0,0546 & 0,2792 & 1,2343 & $-0,1107$ & $2,6893_{\mathrm{a}}$ \\
\hline \multirow[t]{2}{*}{$\beta 23$} & $\begin{array}{c}0,1387 \\
\mathrm{R}^{2}=0,93\end{array}$ & $\begin{array}{l}-1,0256 \mathrm{a} \\
\mathrm{R}^{2}=0,87\end{array}$ & $\begin{array}{r}0,8509_{b} \\
R^{2}=0,77\end{array}$ & $\begin{array}{l}-0,5387 \\
\mathrm{R}^{2}=0,90\end{array}$ & $\begin{array}{l}-2,1489 c \\
R^{2}=0,96\end{array}$ & $\begin{array}{c}-1,7863_{b} \\
\mathrm{R}^{2}=0,95\end{array}$ \\
\hline & $\mathrm{R}^{2} \mathrm{~A}=0,84$ & $\mathrm{R}^{2} \mathrm{~A}=0,69$ & $\mathrm{R}^{2} \mathrm{~A}=0,43$ & $\mathrm{R}^{2} \mathrm{~A}=0,75$ & $\mathrm{R}^{2} \mathrm{~A}=0,90$ & $\mathrm{R}^{2} \mathrm{~A}=0,88$ \\
\hline
\end{tabular}

a : $\alpha \leq 1 \%_{0} ; \mathrm{b}: 1 \%_{0}<\alpha \leq 1 \% ; \mathrm{c}: 1 \%<\alpha \leq 5 \%$.

mination. Après 6 heures d'incubation, à $\mathrm{pH} 5,0,5,6$ ou 6,2 , les pourcentages de germination des spores sont respectivement de 40,94 ou de $98 \%$.

\subsubsection{Sur les cellules végétatives}

Après 24 heures d'incubation, en présence de faibles concentrations en nisine (0-20 UI/ $/ \mathrm{mL})$, le comportement des spores germées dépend essentiellement des valeurs du $\mathrm{pH}$; entre 5,0 et 6,2 , les cellules végétatives, issues des spores germées, se multiplient d'autant plus rapidement que le $\mathrm{pH}$ est proche de la neutralité. Pour des concentrations en nisine plus importantes (20-40 UI/mL), l'inhibition des spores germées, marquée par l'absence de cellules végétatives, dépend du $\mathrm{pH}$ et de la concentration en nisine (figure $1 b$ ). Ainsi, pour des valeurs de $\mathrm{pH}$ supérieures à 5,6, l'inhibition est d'autant plus importante que la concentration en nisine est élevée; pour des valeurs de $\mathrm{pH}$ inférieures à 5,6 et avec $20 \mathrm{UI} / \mathrm{mL}$, l'inhibition augmente avec la diminution du $\mathrm{pH}$. L'activité de la nisine diminue quand la concentration initiale en spores est élevée ; à pH 5,6 , en présence de $10^{5}$ spores $/ \mathrm{mL}, 40$ $\mathrm{UI} / \mathrm{mL}$ de nisine inhibent totalement les spores germées ; cependant, cette concentration est insuffisante lorsque la population initiale est de $10^{6}$ spores $/ \mathrm{mL}$ (résultats non fournis).

\subsection{Combinaison sorbate de potassium / pH}

\subsubsection{Sur les spores}

Le sorbate n'a pas d'action sporicide, mais il perturbe la germination. Pour des valeurs de $\mathrm{pH}$ inférieures à 5,6 , le pourcentage de germination diminue quand la concentration en sorbate aug- 


\begin{tabular}{|c|}
\hline $\begin{array}{c}\text { Facteur fixé } \\
\text { spores }: 10^{5} \mathrm{~mL}\end{array}$ \\
\hline spores/mL \\
\\
$1: 2,0.10^{2}$ \\
$2: 4,1 \cdot 10^{2}$ \\
$3: 8,5 \cdot 10^{2}$ \\
$4: 1,7.10^{3}$ \\
$5: 3,5 \cdot 10^{3}$ \\
$6: 7,1.10^{3}$ \\
$7: 1,4 \cdot 10^{4}$ \\
$8: 2,9 \cdot 10^{4}$ \\
$9: 5,9 \cdot 10^{4}$ \\
$10: 1,2 \cdot 10^{5}$ \\
$11: 2,4 \cdot 10^{5}$ \\
\hline
\end{tabular}

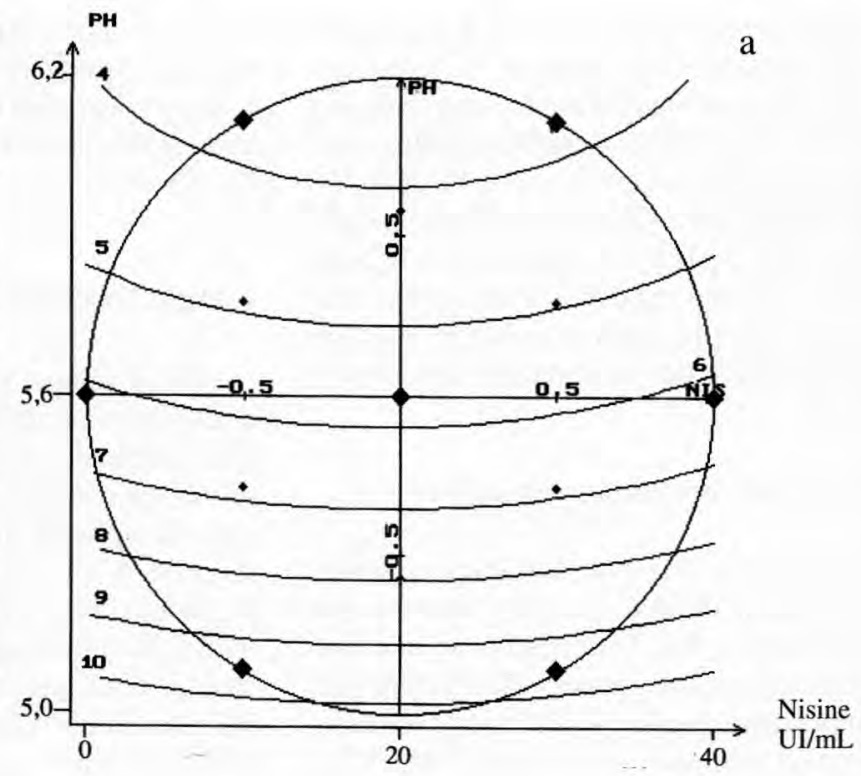

\begin{tabular}{|c|}
\hline $\begin{array}{c}\text { Facteur fixé } \\
\text { spores }: 10^{5} \mathrm{~mL}\end{array}$ \\
\hline cellules \\
végétatives $/ \mathrm{mL}$ \\
$1: 1,0.10^{0}$ \\
$2: 4,6 \cdot 10^{0}$ \\
$3: 2,1 \cdot 10^{1}$ \\
$4: 9,7.10^{1}$ \\
$5: 4,5 \cdot 10^{2}$ \\
$6: 2,0.10^{3}$ \\
$7: 9,5 \cdot 10^{3}$ \\
$8: 4,3 \cdot 10^{4}$ \\
$9: 2,0.10^{5}$ \\
$10: 9,2.10^{5}$ \\
$11: 4,2.10^{6}$ \\
\hline
\end{tabular}

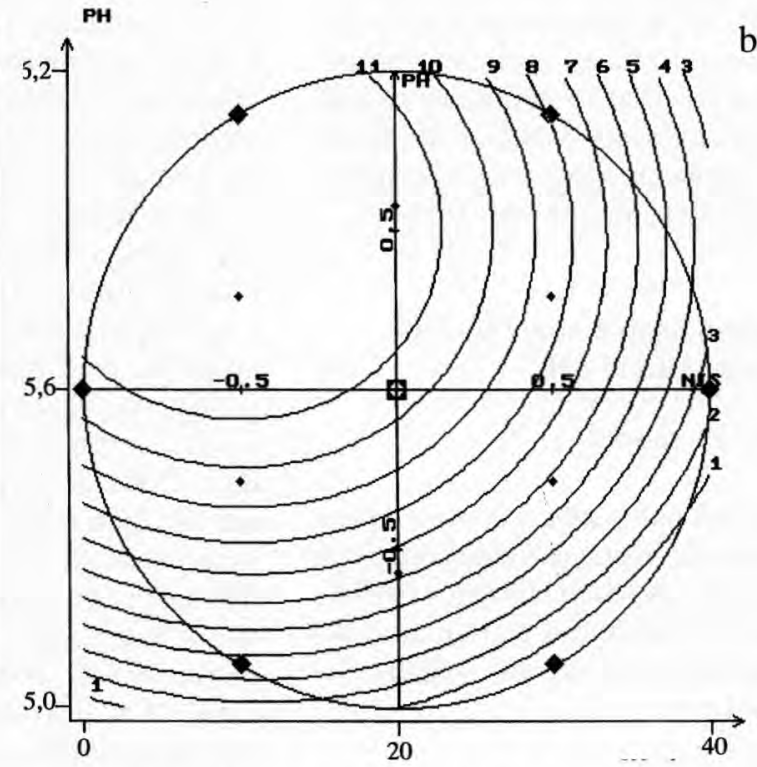

b

Nisine

$\mathrm{UI} / \mathrm{mL}$

Figure 1. Surface de réponse montrant l'effet de la concentration en nisine et du pH sur la croissance de $B$. licheniformis. La concentration en spores est fixée à $10^{5} \mathrm{spores} / \mathrm{mL}$ (valeur au centre du domaine). a. Dénombrement des spores après 6 heures d'incubation à $37^{\circ} \mathrm{C}$. b. Dénombrement des cellules végétatives après 24 heures d'incubation à $37^{\circ} \mathrm{C}$.

Figure 1. Response surface contours showing the effect of nisin concentration and $\mathrm{pH}$ values on the growth of $B$. licheniformis. Spore concentration was fixed at $10^{5} \mathrm{spores} / \mathrm{mL}$ (value at the center of the experimental domain). a. Spore count after $6 \mathrm{~h}$ at $37^{\circ} \mathrm{C}$. b. Vegetative cell count after $24 \mathrm{~h}$ at $37^{\circ} \mathrm{C}$. 
mente (figure $2 a$ ); à pH 5,4 et pour 0 , 0,05 ou $0,15 \%$ de sorbate, les pourcentages de germination sont respectivement de 90,78 ou $53 \%$. Cet effet est plus marqué en milieu acide : en présence de $0,1 \%$ de sorbate, $95 \%$ des spores germent à $\mathrm{pH}$ $5,6,36 \%$ à pH 5,2 et seulement $8 \%$ à $\mathrm{pH}$ 5,0 . L'action inhibitrice du sorbate est faible aux $\mathrm{pH}$ supérieurs à 5,6 , avec un pourcentage de germination supérieur à $96 \%$.

\subsubsection{Sur les cellules végétatives}

Entre 0 et $0,2 \%$ de sorbate, le pouvoir inhibiteur dépend du $\mathrm{pH}$. Pour des $\mathrm{pH}$ inférieurs à 5,6, l'inhibition de la croissance des spores germées est d'autant plus importante que le $\mathrm{pH}$ est acide ; le maximum d'inhibition est observé à $\mathrm{pH} 5,0$ et pour une concentration en sorbate de $0,2 \%$ (figure $2 b$ ). Pour des $\mathrm{pH}$ supérieurs à 5,6 , les concentrations en sorbate retenues n'exercent pas d'effet inhibiteur significatif, car la croissance cellulaire des spores germées s'effectue à une vitesse identique à celle de l'essai témoin sans sorbate.

\subsection{Combinaison nisine / sorbate de potassium / pH}

\subsubsection{Sur les spores}

Les spores ont le même comportement en présence de nisine et/ou de sorbate de potassium. Le pH du milieu est le principal facteur modifiant la germination des spores qui est favorisée aux valeurs élevées de $\mathrm{pH}$.

\subsubsection{Sur les cellules végétatives}

En présence de $0,1 \%$ de sorbate, l'inhibition des spores germées est maximale pour une concentration en nisine de $38 \mathrm{UI} / \mathrm{mL}$ et un $\mathrm{pH}$ proche de 5,7 (figure $3 a$ ). Dans les mêmes conditions expérimentales, l'effet inhibiteur de la nisine ou du sorbate de potassium est faible ; pour une valeur donnée de $\mathrm{pH}$, l'inhibition est d'autant plus importante que les concentrations en sorbate ou en nisine augmentent (figure $3 b$ ).

\section{DISCUSSION}

Afin d'inhiber des flores bactériennes d'altération ou des bactéries pathogènes, des combinaisons judicieuses de bioconservateurs sont une alternative intéressante pour remplacer des conservateurs chimiques. À terme, ces associations complémentaires ou synergiques devraient permettre de diminuer les concentrations de chacun d'eux tout en garantissant la sécurité alimentaire. Cette théorie des barrières [17] a été mise à profit dans différentes études par l'emploi de bactériocines ajoutées ou produites in situ, associées à différents traitements thermiques ou à des conservateurs chimiques [23]. Deux technologies peuvent être utilisées pour réduire les niveaux de contamination en spores dans un aliment ; l'une consiste à empêcher leur germination par leur destruction (stérilisation, rayons X...), l'autre à l'inverse, à favoriser leur germination, mais en présence d'inhibiteurs dont l'action post-germinative doit rester bactéricide jusqu'à la fin de la durée de vie du produit. Cette dernière méthodologie a été retenue dans notre étude. Les spores dormantes de $B$. licheniformis ATCC 14 580 sont placées dans des conditions favorisant leur germination, activation thermique $\left(80^{\circ} \mathrm{C}, 10 \mathrm{~min}\right.$ ) et ajout de L-alanine. La germination des spores, $\mathrm{pH}$-dépendante, diminue aux faibles valeurs de $\mathrm{pH}$ en raison de l'inhibition de la fixation de la L-alanine sur le site d'initiation [6]. La nisine n'est pas sporicide et n'intervient pas lors de la phase initiale de la germination [28]. Son action inhibitrice augmente avec sa concentration et s'exerce sur la phase d'excroissance de la spore. En général, cette activité est faible 

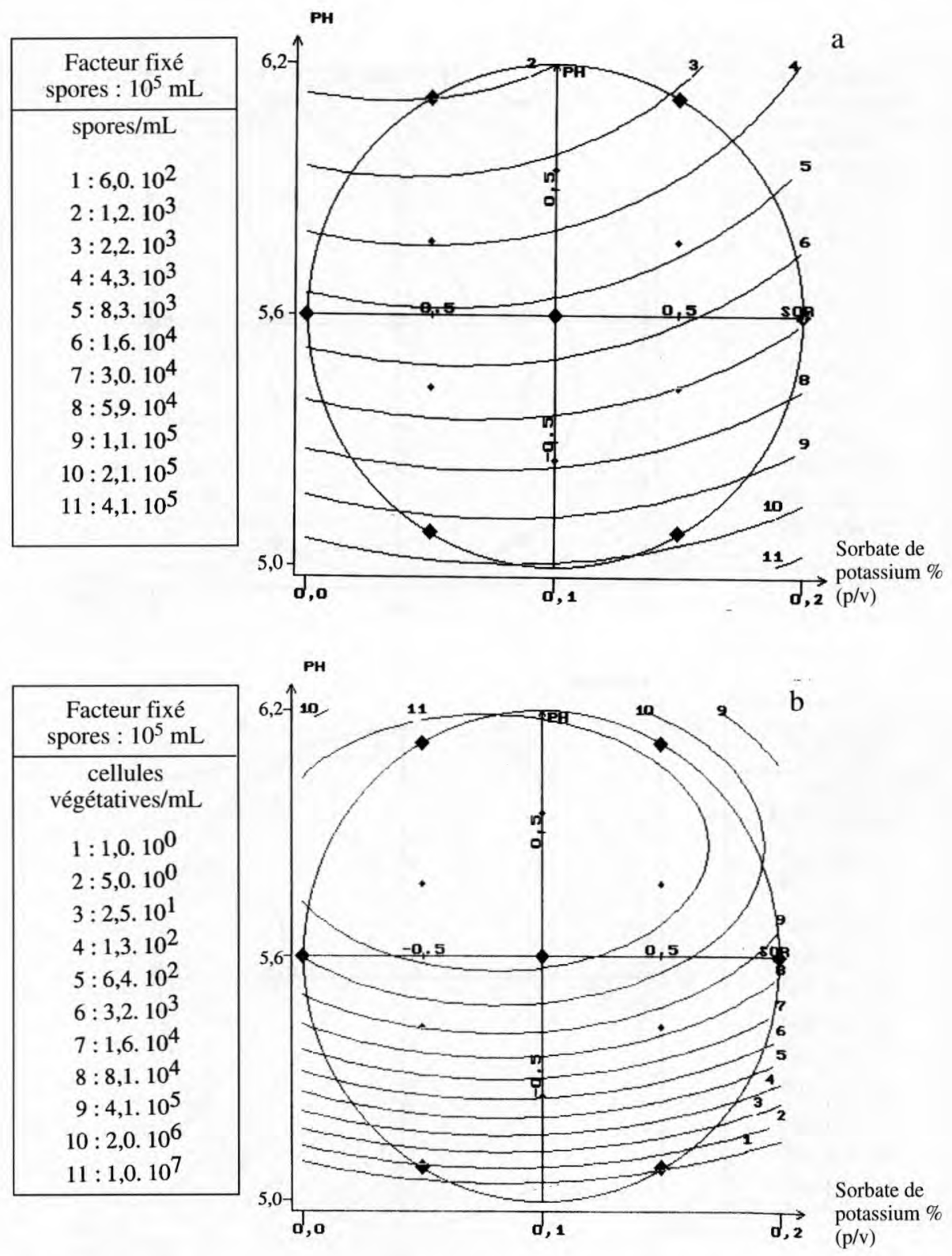

Figure 2. Surface de réponse montrant l'effet de la concentration en sorbate et du pH sur la croissance de B. licheniformis. La concentration en spores est fixée à $10^{5}$ spores $/ \mathrm{mL}$ (valeur au centre du domaine). a. Spores après 6 heures d'incubation à $37^{\circ} \mathrm{C}$. b. Cellules végétatives après 24 heures d'incubation à $37^{\circ} \mathrm{C}$.

Figure 2. Response surface contours showing the effect of potassium sorbate concentration and $\mathrm{pH}$ values on the growth of $B$. licheniformis. Spore concentration was fixed at $10^{5} \mathrm{spores} / \mathrm{mL}$ (value at the center of the experimental domain). a. Spore count after $6 \mathrm{~h}$ at $37^{\circ} \mathrm{C}$. b. Vegetative cell count after $24 \mathrm{~h}$ at $37^{\circ} \mathrm{C}$. 

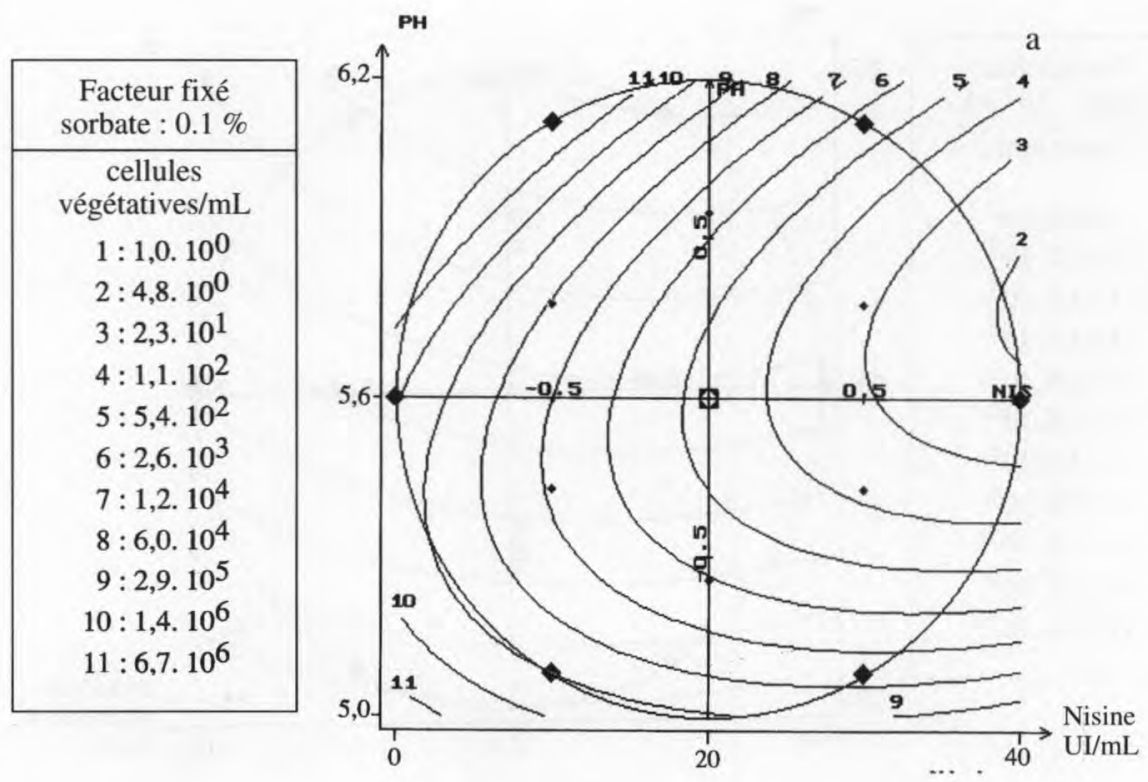

\begin{tabular}{|c|}
\hline Facteur fixé \\
$\mathrm{pH}: 5.7$ \\
\hline cellules \\
végétatives/mL \\
$1: 1,0.10^{0}$ \\
$2: 4,8.10^{0}$ \\
$3: 2,3 \cdot 10^{1}$ \\
$4: 1,1 \cdot 10^{2}$ \\
$5: 5,4 \cdot 10^{2}$ \\
$6: 2,6 \cdot 10^{3}$ \\
$7: 1,2.10^{4}$ \\
$8: 6,0.10^{4}$ \\
$9: 2,9.10^{5}$ \\
$10: 1,4 \cdot 10^{6}$ \\
$11: 6,7.10^{6}$ \\
\hline
\end{tabular}

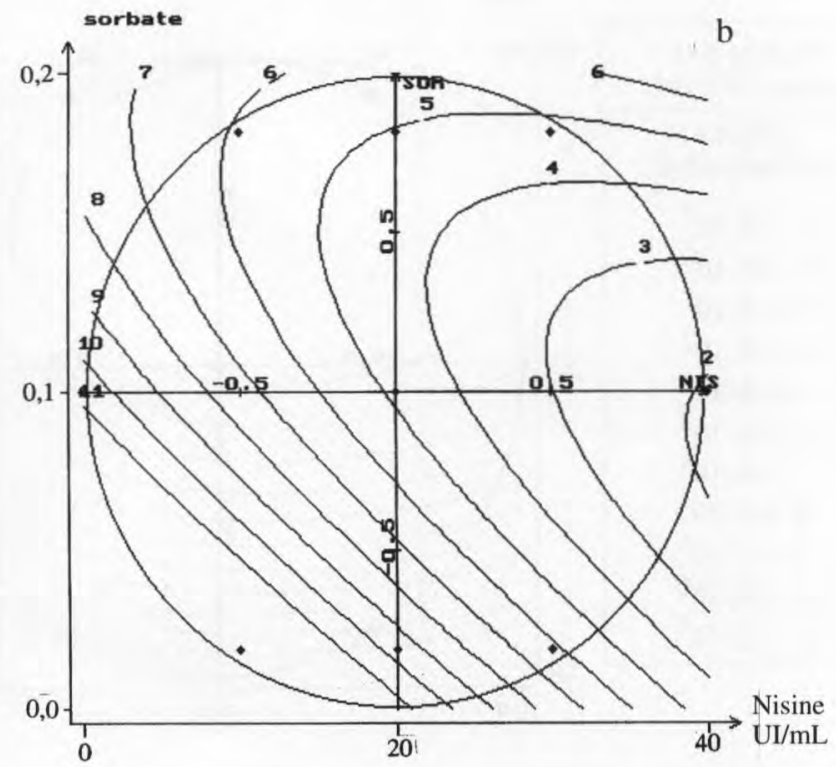

Figure 3. Surface de réponse indiquant le dénombrement des cellules végétatives de $B$. licheniformis après 24 heures d'incubation à $37^{\circ} \mathrm{C}$. a. En fonction de la concentration en nisine et du pH (sorbate : $0,1 \%(\mathrm{p} / \mathrm{v})$. b. En fonction des concentrations en nisine et en sorbate de potassium $(\mathrm{pH}: 5,7)$.

Figure 3. Response surface contours of B. licheniformis vegetative cell count after $24 \mathrm{~h}$ at $37^{\circ} \mathrm{C}$. a. As a function of nisin concentration and $\mathrm{pH}$ values (potassium sorbate: $0.1 \%(\mathrm{w} / \mathrm{v})$ ). $\mathbf{b}$. As a function of nisin and potassium sorbate concentrations ( $\mathrm{pH}: 5.7)$. 
à $\mathrm{pH}[13,15]$ et niveaux en spores élevées [22]. Sa solubilité et sa stabilité diminuent quand le $\mathrm{pH}$ augmente. La nisine augmente la phase de latence, mais lorsque la croissance des spores germées est initiée, le taux de multiplication reste identique à celui du témoin [4]. Le sorbate de potassium, non sporicide, perturbe la germination voire l'inhibe à des concentrations élevées. Des concentrations entre 0,1 et $0,2 \%(\mathrm{p} / \mathrm{v})$ exercent un effet répressif sur la germination, d'autant plus marqué que sa concentration est importante. Ce composé agit comme un agent compétitif de la L-alanine pour le site d'initiation de la germination [24]. Les inhibitions de la germination de la spore et de l'excroissance dépendent à la fois de la concentration en sorbate et du $\mathrm{pH}$ du milieu. $\mathrm{A} \mathrm{pH}$ bas, elle est en relation avec les formes non dissociées du sorbate de potassium ( $\mathrm{pKa}=4,75$ ) et de l'acide lactique ( $\mathrm{pKa}$ $=3,85$ ) $[25]$. Aux concentrations préconisées, l'efficacité inhibitrice du sorbate est généralement faible, mais elle augmente à $\mathrm{pH}$ acide et aux concentrations élevées. La concentration en sorbate autorisée dans les fromages ne doit pas dépasser $0,2 \%$, selon la réglementation française [8]. Nos résultats, obtenus avec les spores de $B$. licheniformis dans du lait acidifié, indiquent que $0,2 \%$ de sorbate de potassium est une concentration létale à $\mathrm{pH} 5,0$, mais insuffisante aux valeurs de $\mathrm{pH}$ supérieures.

Les associations sorbate de potassium, nisine, acide lactique permettent dans certains cas d'observer des complémentarités d'actions inhibitrices. Ces résultats offrent la possibilité d'associer la nisine et le sorbate de potassium, puisque l'effet inhibiteur maximal est observé aux $\mathrm{pH}$ proches de la neutralité. Ces inhibiteurs employés seuls, aux mêmes concentrations, ne sont pas bactéricides. Des recherches concernant l'association de la nisine et du sorbate de potassium, composés GRAS, dans du lait acidifié, sont rarement rapportées.
Des combinaisons appropriées de ces bioconservateurs mériteraient d'être davantage envisagées, en raison de leur innocuité pour la santé humaine. Ces résultats pourraient trouver à terme des applications pratiques pour la maîtrise de la croissance de flores bactériennes indésirables, en combinaison avec de bonnes pratiques de fabrication.

\section{RÉFÉRENCES}

[1] An-Erl King V., Studies on the control of the growth of Saccharomyces cerevisiae by using response surface methodology to achieve effective preservation at high water activities, Int. J. Food. Sci. Technol. 28 (1993) 519-529.

[2] Avery S.M., Hudson J.A., Phillips D.M., Use of response surface models to predict bacterial growth from time/temperature histories, Food. Control. 7 (1996) 121-128.

[3] Banks J.G., Morgan S., Stringer M.F., Inhibition of heated Bacillus spores by combinations of potassium sorbate, sodium benzoate, $\mathrm{pH}$ and organic acids, Lebensm. Wiss. u. Technol. 21 (1988) 250-255.

[4] Bell R.G., De Lacy K.M., The efficacy of nisin, sorbic acid and monolaurin as preservatives in pasteurised cured meat products, Food Microbiol. 4 (1987) 277-283.

[5] Bergère J.L., Cerf O., Les bactéries sporulées, In : Les groupes microbiens d'intérêt laitier, CEPIL, Nancy, 1992

[6] Blocher J.C., Busta F.F., Inhibition of germinant binding by bacterial spores in acidic environments, Appl. Environ. Microbiol. 50 (1985) 274-279.

[7] Crielly E.M., Logan N.A., Anderton A., Studies on the Bacillus flora of milk and milk products, J. Appl. Bacteriol. 77 (1994) 256-263.

[8] Dacosta Y., L'acide sorbique et les sorbates, Tec et Doc Lavoisier, Paris, 1994

[9] Doehlert D.H., Uniform shell designs, Appl. Statistics 19 (1970) 231-239.

[10] Estavoyer J,M., Meyer V, Leroy J., Vanlemmens P., Plesiat P., Balvay P., Dupont M.J., Michel-Briand Y., Méningite à Bacillus licheniformis, Ann. Med. Intern 146 (1995).

[11] Hirsch A., Growth and nisin production of a strain of Streptococcus lactis, J. Gen. Microbiol. 5 (1951) 208. 
[12] Hull R., Toyne S., Haynes I., Lehmann F., Thermoduric bacteria : a reemerging problem in cheese making, Aust. J. Dairy Technol. 47 (1992) 91-94.

[13] Hurst A., Hoover D.G. Nisin, In : Davidson P.M., Branen A.L. (Eds.), Antimicrobials in foods, New York, 1993

[14] Irie R., Okamoto T., Fujita Y., Kinetics of spore germination of Bacillus subtilis in low concentrations of L-alanine, J. Gen. Appl. Microbiol. 30 (1984) 109-113.

[15] Kumar N., Prasad D.N., Factors affecting the inhibitory action of nisin against Lactobacillus and Bacillus species, M. A. N. 12 (1994) 155-161.

[16] Larpent J.P., Larpent-Gourgaud M., Manuel pratique de microbiologie, Hermann, Paris, 1985

[17] Leistner L., Gorris L.G.M., Food preservation by hurdle technology, Trends Food Sci. Tech. 6 (1995) 41-46.

[18] Liewen M.B., Marth E.H., Growth and inhibition of microorganisms in the presence of sorbic acid: a review, J. Food Prot. 48 (1985) 364-375.

[19] Mathieu D., Phan-Tan-Luu R., New efficient methodology for research using optimal design, LPRAI université d'Aix-Marseille, 1992

[20] McClintock M., Serres L., Marzolf J.J., Hirsch A., Mocquot G., Action inhibitrice des streptocoques producteurs de nisine sur le développement des sporulés anérobies dans le fromage de gruyère fondu, J. Dairy Res. 19 (1952) 187-193.
[21] Oleyede O.B., Scholefield J., Inhibition of Bacillus spores by combinations of heat, potassium sorbate, $\mathrm{NaCl}$ and $\mathrm{pH}$, World $\mathbf{~}$. Microbiol. Biotechnol. 10 (1994) 579-582.

[22] Oscroft C.A., Banks J.G., Mc Phee S., Inhibition of thermally-stressed Bacillus spores by combinations of nisin, $\mathrm{pH}$ and organic acids, Lebensm. Wiss. u. Technol. 23 (1990) 538-544.

[23] Rayman M.K., Aris B., Hurst A., Nisin: a possible alternative or adjunct to nitrite in the preservation of meats, Appl. Environ. Microbiol. 41 (1981) 375-380.

[24] Seward R.A., Deibel R.H., Lindsay R., Effects of potassium sorbate and other antibotulinal agents on germination and outgrowth of Clostridium botulinum type E spores in microcultures, Appl. Environ. Microbiol. 44 (1982) 1212-1221

[25] Sofos J.N., Pierson M.D., Blocher J.C., Busta F.F., Mode of action of sorbic acid on bacterial cells and spores, Int. J. Food Microbiol. 3 (1986) 1-17.

[26] Sofos J.N., Busta F.F., Sorbic acid and sorbates. In: Davidson P.M., Branen A.L. (Eds.), Antimicrobials in foods, New York, 1993

[27] Van Heddeghem A., Vlaemynck, Sources of contamination of milk with Bacillus cereus on the farm and in the factory, Bull. Int. Dairy Fed. 275 (1992) 19-22.

[28] Venema K., Venema G., Kok J., Lactococcal bacteriocins: mode of action and immunity, Trends Microbiol. 3 (1995) 299-304. 